\title{
O discurso científico/colonialista norte-americano sobre Xuxa
}

\section{The North American Scientific/Colonialist Discourse about Xuxa}

Fernando Zolin-Vesz*

Instituto Federal de Educação, Ciência e Tecnologia de Mato Grosso Cuiabá - Mato Grosso / Brasil

RESUMO: O objetivo deste artigo é analisar o livro Xuxa: The Mega-Marketing of Gender, Race, and Modernity, de Amelia Simpson, publicado em 1993, ainda uma das principais referências para aqueles que buscam compreender o fenômeno Xuxa. Tendo como premissa o apagamento de qualquer ideal de neutralidade e objetividade na produção de conhecimento, os resultados da análise apontam para a reprodução de um discurso colonialista ao descrever o fenômeno como típico de um país pertencente ao terceiro mundo - subdesenvolvido, com uma população que apresenta baixo índice de escolaridade e que é facilmente manipulável pelos meios de comunicação.

PALAVRAS-CHAVE: Produção de conhecimento; discurso colonialista; fenômeno Xuxa.

ABSTRACT: The purpose of this paper is to analyze 1993 Amelia Simpson's book Xuxa: The Mega-Marketing of Gender, Race, and Modernity, still one of the main references to those who are in search of comprehending the phenomenon Xuxa. Based on the premise of the abolishment of any ideal of neutrality and objectivity in the production of knowledge, the results of the analysis point to the reproduction of a colonialist discourse by describing the phenomenon like a typical one come from a third world country - underdeveloped, with an undereducated population that is easily manipulated by mass media.

KEYWORDS: Production of knowledge; colonialist discourse; phenomenon Xuxa.

O papel do intelectual não é mais o de se colocar "um pouco na frente ou um pouco de lado" para dizer a muda verdade de todos; é antes o de lutar contra as formas de poder exatamente onde ele é, ao mesmo tempo, o objeto e o instrumento: na ordem do saber, da "verdade", da "consciência", do discurso.

Michel Foucault

* fernando.vesz@blv.ifmt.edu.br 
Desde que o foco de seu trabalho passou a ser o universo infantil, em meados da década de 1980, Maria da Graça Xuxa Meneghel se transformou em um dos maiores ícones da televisão, da indústria fonográfica e do cinema no Brasil. O sucesso lhe rendeu a designação de "Rainha dos Baixinhos", e seu nome se tornou conhecido internacionalmente.

No início da década de 1990, versōes em espanhol de seus programas infantis eram exibidas por várias emissoras de televisão na América Latina, na Espanha e para o público hispânico dos Estados Unidos. Igualmente, versões em espanhol de seus discos registravam alto índice de venda. Essa notoriedade lhe dispensou também o convite para apresentar, em 1993, um programa infantil em inglês, exibido por mais de cem canais independentes de televisão nos Estados Unidos (VALDIVIA; CURRY, 1998; 2000; RASHKIN, 1999; CURRY; VALDIVIA, 2002).

Sua notoriedade conduziu, então, pesquisadores norte-americanos dos estudos culturais e da mídia a analisar e interpretar o fenômeno Xuxa como um ícone da comunicação de massa que chegava a milhōes de pessoas na América Latina, nos Estados Unidos e em parte da Europa. O pioneiro e principal trabalho nesse campo de investigação foi o livro Xuxa: The Mega-Marketing of Gender, Race, and Modernity, de Amelia Simpson, publicado em 1993. Elissa Rashkin (1999) considera que Xuxa foi apresentada aos acadêmicos norte-americanos por meio do estudo de Simpson. Já Angharad N. Valdivia e Ramona Curry (2000) consideram Simpson a única pesquisadora norte-americana a pesquisar criticamente Xuxa como um ícone cultural brasileiro.

Apesar das críticas dessas autoras em relação à análise de Simpson, para quem "a predominante análise do estrelato de Xuxa [...] corre o risco de oferecer explicações personalizadas sobre um ícone individual ignorando os poderosos processos da mídia internacional que delineiam e disseminam o ícone e seu impacto popular" (VALDIVIA; CURRY, 2000, p. 127-128), ${ }^{1}$ o estudo de Simpson continua sendo germinal, sobretudo para aqueles que se debruçam em compreender o fenômeno Xuxa, constituindo uma das principais referências, tanto nas pesquisas das autoras encartadas no parágrafo anterior quanto para as escassas pesquisas nacionais que buscam compreender o fenômeno Xuxa no Brasil - veja, por exemplo, Vanessa Patricia M. Campos (2006), Maria Julianna F. M. Sinval (2010) e Fernando Zolin-Vesz e Dánie Marcelo de Jesus (2012).

${ }^{1}$ Todas as traduções são de minha autoria. 
Neste artigo, intento analisar o estudo de Simpson, tendo como premissa o apagamento de qualquer ideal de neutralidade e objetividade na produção de conhecimento (MOITA LOPES, 2004; 2006; 2009), ou seja, que o pesquisador constrói e produz significados sobre o que estuda calcado em sua sócio-história. Dessa forma, compreendo que, para além da análise de um ícone da comunicação de massa brasileiro, que "construiu um império em torno de um programa de televisão destinado a crianças" também "transmitido em dezesseis países da América Latina bem como nos Estados Unidos - no canal de língua espanhola Univisión", cuja "versão em língua inglesa do programa está prevista para ser exibida nos Estados Unidos em 1993" (SIMPSON, 1993, p. 2), a análise proposta no livro de Simpson entreabre determinadas representações sobre o fenômeno Xuxa e sobre o Brasil que podem ser associadas a um discurso colonialista, ao (des)classificar o fenômeno como único, típico de um país do terceiro mundo, também "único no mundo de hoje" (SIMPSON, 1993, p. 2).

Em se tratando da principal referência, ou mesmo única - como observam Valdivia e Curry (2000) - que ainda hoje temos para compreender o mito Xuxa, os significados que o estudo apresenta são reproduzidos como "verdades científicas" aparentemente perpetuadoras de um discurso colonialista que, há mais de quinhentos anos, rotula os povos dos países latino-americanos (não esquecidas suas manifestações culturais) como inferiores, subdesenvolvidos, atrasados. Quando alguma dessas manifestações se torna mais ou menos notória, é vista apenas como uma "curiosidade estrangeira" (RASHKIN, 1999, p. 205).

\section{$O$ apagamento de qualquer ideal de neutralidade e objetividade na produção de conhecimento}

De acordo com Luiz Paulo da Moita Lopes (2004; 2006; 2009), estamos vivendo tempos em que aflora profundo questionamento dos modos tradicionais de fazer pesquisa nas Ciências Sociais e Humanas. Dentro desse espectro, o autor questiona como seria possível ainda falar de verdades científicas após Michel Foucault (em 1979) ter apresentado a relação entre verdade e poder, ponto-chave para a compreensão do que o autor denomina de apagamento de qualquer ideal de neutralidade e objetividade na produção de conhecimento.

Para Foucault "a verdade não existe fora do poder ou sem poder", haja vista que cada sociedade possui seu "regime de verdade", ou seja, 
os tipos de discurso que ela acolhe e faz funcionar como verdadeiros; os mecanismos e as instâncias que permitem distinguir os enunciados verdadeiros dos falsos, a maneira como se sanciona uns e outros; as técnicas e os procedimentos que são valorizados para a obtenção da verdade; o estatuto daqueles que têm o encargo de dizer o que funciona como verdadeiro. (FOUCAULT, 2012 [1979], p. 12).

Dessa forma, ainda na trilha do mesmo autor, a relação entre verdade e poder em nossas sociedades assume cinco características historicamente construídas, das quais destaco (a) a "verdade" é centrada na forma do discurso científico e nas instituições que o produzem; e (b) a "verdade" é produzida e transmitida sob o controle dominante de alguns grandes aparelhos políticos ou econômicos, como a universidade e os meios de comunicação (FOUCAULT, 2012 [1979], p. 13). O intelectual, portanto, não é o "portador de valores universais", mas "alguém que ocupa uma posição específica”, cuja particularidade está ligada principalmente à "política de verdade nas sociedades contemporâneas" (FOUCAULT, 2012 [1979], p. 13).

Sendo assim, conforme o autor, há um combate "em torno do estatuto da verdade e do papel econômico-político que ela desempenha” (FOUCAULT, 2012 [1979], p. 13). Verdade, segundo o autor, refere-se ao "conjunto das regras segundo as quais se distingue o verdadeiro do falso e se atribui ao verdadeiro efeitos específicos de poder" (FOUCAULT, 2012 [1979], p. 13). Por isso, a relação entre verdade e poder, na produção de conhecimento, é apontada por Foucault como o problema político essencial para o intelectual. O problema não seria "mudar a consciência das pessoas [...] mas o regime político, econômico, institucional de produção da verdade”, ou seja, "desvincular o poder da verdade das formas de hegemonia (sociais, econômicas, culturais) no interior das quais ela funciona no momento" (FOUCAULT, 2012 [1979], p. 14).

Em suma, Foucault propõe profundos questionamentos sobre os tipos de conhecimento construído e a que propósitos servem, evidenciando, nessa marcha, o apagamento de qualquer ideal de neutralidade e objetividade na produção de conhecimento.

\section{O discurso colonial - "nós" versus "eles"}

À luz do pensamento de Ania Loomba (2005), a obra Orientalismo, de Edward Said (1978), inaugura outro tipo de estudo sobre o colonialismo. A premissa foucaultiana de que o conhecimento não é inocente, mas está 
profundamente conectado com a relação verdade-poder, constitui o fio teórico do livro que, segundo a autora, não cuidava de culturas não ocidentais, antes se aplicava às representações do Ocidente sobre essas culturas. Assim, essas representaçôes contribuíram para a criação de uma dicotomia entre a Europa e seus "outros", central para a manutenção e expansão da hegemonia europeia sobre outros territórios. O estudo do Oriente, portanto, não era objetivo, mas "uma visão política da realidade cuja estrutura promovia a diferença entre o familiar (a Europa, o Oeste, 'nós') e o estranho (o Oriente, o Leste, 'eles')" (SAID, 1978, p. 45).

Os estudos sobre discurso colonial, ainda à luz do pensamento da mesma autora, indicam nova forma de conceber a interação dos processos cultural, intelectual, econômico e político na formação, perpetuação e desmembramento do colonialismo, ao examinar a intersecção entre ideias e instituiçôes, conhecimento e poder. Sendo assim, esses estudos buscam investigar como estereótipos, imagens e "conhecimento" sobre sujeitos e culturas estão imbricados com instituições de controle econômico, administrativo, científico etc.

Nessa seara, conforme Sara Mills (2004), os povos colonizados eram desumanizados por meio de generalizaçôes - uma massa indistinguível sobre a qual se poderia coletar "conhecimento" ou que poderia ser estereotipada-, o dócil hindu e o insensível chinês, por exemplo. Loomba (2005) assegura perceber esse mesmo processo de generalização em relação tanto aos árabes muçulmanos, após os ataques ao World Trade Center e ao Pentágono, nos Estados Unidos, em 11 de setembro de 2001 - vistos como culturalmente condicionados a serem violentos - quanto aos asiáticos - vistos como diligentes, mas fortemente ligados às próprias regras sobre negócios e família. Ambas as generalizações, observa Loomba (2005), consideram essas culturas não condizentes com o mundo ocidental. A descrição do "outro", portanto, é geralmente em termos negativos. Essa negatividade, no pensar de Mills (2004), é uma característica discursiva produzida dentro do contexto colonial que constituía as estruturas discursivas para produzir "conhecimento" e "verdades científicas".

\section{Xuxa: fenômeno único, oriundo de país também único}

Como pode ser observado já no subtítulo do livro de Simpson, sua análise gira em torno de três eixos: gênero (gender), raça (race) e modernidade (modernity). Seguindo-lhe os passos, a análise "examina aspectos da cultura brasileira que estão refletidos nas características definidoras do estrelato de 
Xuxa: sua representação de feminilidade [gênero], sua exclusiva beleza branca [raça] e a promoção de uma ideia de cultura e modernidade baseada no consumismo" (SIMPSON, 1993, p. 10-11).

O livro está organizado em cinco capítulos, não esquecida a introdução. O primeiro, denominado "Myths of Beauty and Myths of Race", segundo a autora, mostra como Xuxa despontou na televisão em 1983 com um conjunto de associações que remetem a configurações hegemônicas sobre gênero e raça no Brasil. O segundo capítulo, com o título de "Xuxavision: Programmed Euphoria", examina a imagem de Xuxa na televisão, desde seu período na TV Manchete (1983-1985) até o período do programa Xou da Xuxa na TV Globo (1986-1992). Patenteia como a fórmula de seus programas, aliada ao que a autora chama de "xuxacentrismo" (Xuxacentric design), contribui para reforçar atitudes e valores hegemônicos. Já o terceiro capítulo, "Mass Marketing the Messages", analisa como o incentivo de Xuxa ao consumismo representa a expressão fundamental da devoção à apresentadora, e como suas músicas, seus filmes e seus diversos produtos informam a visão projetada de Xuxa sobre o Brasil. O quarto capítulo, designado "Xhaping the Future", analisa os "clones" de Xuxa - a autora cita a apresentadora Angélica Ksyvickis como a mais bem-sucedida entre suas diversas imitações - e os lugares do fenômeno Xuxa, em diversas perspectivas. Isso permite identificar alguns de seus sentidos figurativos para o futuro de um Brasil inscrito em um modelo de cultura agora dirigido pela mídia. Por fim, o quinto capítulo, "Kids and Kidnappers", investiga criticamente um dos eventos, na história de Xuxa, considerado pela autora "grande virada" em sua narrativa - a tentativa de sequestro da apresentadora e uma das paquitas nos idos de 1991. Para a autora, o episódio encetou um debate nacional sobre a ideia que o país tinha de si, o que contribuiu para a expansão do império de Xuxa para além das fronteiras nacionais.

Nesse ínterim, inicio a análise a partir da descrição de Xuxa como um fenômeno único que Simpson, implicitamente, parece propor. Essa unicidade é representada, no entender da autora, a partir do consenso de aprovação entre o público em geral, como um dos mais interessantes aspectos do fenômeno Xuxa, ou seja, "a onipresente imagem de Xuxa é raramente questionada no discurso público brasileiro" (SIMPSON, 1993, p. 9-10). A autora retoma o início da carreira de Xuxa, principalmente sua participação no filme Amor, estranho amor (1982) e os diversos ensaios nus para revistas, como a Playboy, para exemplificar a unicidade do fenômeno Xuxa. 
While Xuxa's performance with the boy in Amor Estranho Amor attracted attention in Brazil after she became a children's star, she was not censured for it. Her role in the film and her nude modeling in the early eighties seemed to make Xuxa, if anything, more likable [...] Xuxa's appearance in the soft-porn movie, rather than provoking fears that she might inspire a precocious sexuality in children or incite child-centered sexual fantasies in adults, became part of the image of femininity that endears her to audience by conforming to, and thus affirming, the validity of familiar gender roles. (SIMPSON, 1993, p. 30-31).

Como assegura a autora, a participação de Xuxa no filme, mesmo depois de ter se tornado estrela dos programas infantis, não foi censurada nem provocou receios de que poderia incentivar a sexualidade precoce nas crianças, quando não incitar fantasias sexuais com crianças por parte dos adultos. Ao contrário, prossegue a autora, sua participação no filme se tornou parte da imagem de feminilidade que a faz admirada e amada pelo público, já que está de acordo e afirma a validade dos papéis de gênero. Entretanto, essa imagem ecoa traços de parâmetros de feminilidade que a autora acredita ser possível identificar também ao redor do mundo.

Xuxa's image registers a history of attitudes about gender and race that are not unique to Brazil. These attitudes find expression the world over in a variety of manifestations. In the United States, for example, the Barbie doll, originally a sex toy for adult men, echoes in the imaginations of generations of children. The typical Barbie look is blond and white [...] Yet that representation of ideal femininity, like Xuxa's, has thrived in the promotional environment of consumer culture. Cy Schneider, the man responsible for launching Barbie's ad campaign on television, understood how the doll worked: "Little girls saw Barbie as the young woman they wanted to be someday". The ideals Barbie and Xuxa project, of course, are so far beyond reach that the fundamental lesson learned is about not measuring up. Barbie and Xuxa are icons that play on and feed back a social definition of feminine beauty and perfection. Through television's relentless marketing, Barbie became "the most popular toy in history", and Xuxa emerged as Brazil's "Queen of Kids" (SIMPSON, 1993, p. 9).

A partir da comparação com a boneca Barbie, Simpson busca identificar a definição de beleza feminina e perfeição que parece ancorar a imagem de feminilidade que a autora também observa em sua análise sobre o fenômeno Xuxa - ser loira e branca. Barbie e Xuxa são, portanto, ícones dessa 
representação - a boneca se tornou o brinquedo mais popular da história, e Xuxa ascendeu ao pódio de "Rainha dos Baixinhos" brasileiros.

Entretanto, ao contrário do Brasil, em que há consenso de aprovação sobre Xuxa, nos Estados Unidos perdura alguma resistência em relação ao papel da boneca Barbie como ícone de feminilidade.

There is some resistance in the United States to Barbie's function as a fashion icon selling images of femininity in the form of commodities and sexualizing the body in the interests of marketability. Susan Faludi tells of a young feminist, for example, who defines herself with the slogan "I am not a Barbie doll". (SIMPSON, 1993, p. 9).

Como é possível perceber, a unicidade do fenômeno Xuxa, na análise de Simpson, se dá a partir da relação "nós" versus "eles". A despeito de a representação de feminilidade, presente na imagem de Xuxa, enfeixar conotações universais, como a própria autora assinala, Simpson parece reproduzir um discurso colonialista que vê o "outro" como único, já que, no caso do fenômeno Xuxa ("eles"), ao contrário da boneca Barbie nos Estados Unidos ("nós"), não se opõe resistência em relação às formas de comodificação e sexualização do corpo. Muito pelo contrário, o que a autora observa é o consenso, a naturalização.

Todavia, esse processo só é possível porque oriundo de um país também único - o Brasil. A bem dizer, ainda nas pegadas da autora, o fenômeno Xuxa reflete aspectos da cultura brasileira que, associados a uma indústria dos meios de comunicação de massa superdesenvolvida, tornam o país um caso único.

The ideological impact of the entertainment Xuxa provides is all the more powerful for being projected by the sophisticated Globo network at a population more likely to have a television set than a refrigerator or running water. Brazil may be unique in the world today in its peculiar combination of a highly developed mass communications industry and an undereducated, needy population that nevertheless watches a lot of television. Brazilians, in fact, are said to watch more television than any other people of the third world. (SIMPSON, 1993, p. 2).

Apropriado, parafraseando a autora, pontuar que a unicidade do Brasil se refere a uma "peculiar combinação" entre essa indústria dos meios de comunicação de massa e uma população necessitada, titular de baixo índice de escolaridade, mas a que mais assiste à televisão no terceiro mundo. Como 
enfatiza a autora, a população brasileira se faz mais propícia a ter um aparelho de televisão do que uma geladeira ou água encanada.

Nesse andar, a unicidade do Brasil é construída pela autora também assentada na comparação entre "nós" versus "eles", registrando que, neste caso, "nós" se refere aos países do primeiro mundo, e "eles" alude aos países do terceiro mundo, a exemplo do Brasil. Nessa relação, um dos aspectos que a autora pontua diz respeito ao número de aparelhos de televisão. Simpson afirma que o Brasil pode ser comparado a países do primeiro mundo, ocupando, com 28 milhões de aparelhos, o quarto lugar no ranking mundial.

The media's intrusion into the lives of Brazilians is comparable to that in many first world countries: Bazilians watch more television than any other third world people. The nation ranks fourth in the world in the number of television sets (28 million), behind only the United States (154 million), Japan (79 million), and Britain (33 million). A study of television viewing in Brazil's largest city, São Paulo, showed that 95 percent of the population watch regularly during the week. Ninety percent watch television regularly during the week, and 26 percent of those are heavy viewers. (SIMPSON, 1993, p. 43).

Entretanto, como assinala a autora, há uma correlação entre as classes sociais que mais assistem à televisão, regularmente, e aquelas com baixo índice de escolaridade e baixos salários - "interestingly, there is a correlation between heavy television viewing and low educational levels, and there are indications of a connection with low income levels" (SIMPSON, 1993, p. 43). Dessa forma, a autora argumenta que, diante da crescente influência dos meios de comunicação de massa na América Latina a partir da década de 1980, padronizando a informação, os valores sociais, as formas de beleza e as tendências de consumo, as expectativas são mais propensas a serem frustradas no Brasil do que nos países do primeiro mundo. Simpson considera que a capacidade de consumo dos brasileiros, não diversamente à capacidade de corresponder aos ideais projetados pela mídia, é decepcionante.

The decade of the eighties in Latin America was one of economic decline and, at the same time, one of tremendous growth in the influence of mass communications. The eighties saw what professors Regina Festa and Luiz Fernando Santoro call the development through the media of a "vast network of unprecedent social standardization through which passed, on a massive scale, information, social values, aesthetic forms, consumer trends, and the systematic and cumulative 
construction of symbolic modes and social relations". One of the effects of this process was to raise expectations that were much more likely to be frustrated in Brazil than in the similarly media saturated first world. The average Brazilian's ability to purchase goods or to conform to the ideals projected by the media mass is disappointing. Nico Vink writes that "a contradictory consumption society has been formed in which, for the majority of the population, the images of consumption goods have increased more than the real access to these goods". The media cut across class lines to offer the same dream of happiness - through the purchase of a refrigerator, a pork roast, or a pair of Xuxa sandals - to the rich who can buy them all and to the poor who can afford none. (SIMPSON, 1993, p. 43-44).

A comparação entre o Brasil e os países do primeiro mundo enfoca, portanto, o eixo central da análise de Simpson. Para tanto, a autora recorre a generalizações, dado que a população brasileira desfila baixo índice de escolaridade e é mais propícia a ter um aparelho de televisão do que uma geladeira ou água encanada. Soma-se ainda a capacidade de os brasileiros corresponderem aos ideais projetados pela mídia ser decepcionante por força dos baixos salários. Assentada nisso, Simpson apresenta uma imagem negativa do Brasil em comparação com os países do primeiro mundo. Bem essas imagens negativas, em sua conclusão, tornam o Brasil um país único. De certa forma, é como se a autora, implicitamente, patrocinasse que a apresentadora Xuxa apenas se transformou em um fenômeno da mídia porque o início de sua carreira se deu em um país detentor de uma indústria dos meios de comunicação de massa superdesenvolvida, somado a isso o fato de o país corresponder à população que mais assiste à televisão no terceiro mundo. Ao mesmo tempo, essa população, marcada por baixo índice de escolaridade e por baixos salários, conduz a autora a visualizar tal fato como relevante para o sucesso da televisão nos países do terceiro mundo.

A unicidade do fenômeno Xuxa, promovida pela análise de Simpson, parece, em meu modo de ver, ecoar a premissa foucaultiana de que o conhecimento não é inocente, antes está profundamente conectado com a relação verdade-poder. Nesse viés, as imagens negativas que a autora entremostra sobre o fenômeno Xuxa contribuem para reproduzir, parafraseando Said (1978), uma visão política da realidade cuja estrutura promove a diferença entre o familiar (os Estados Unidos, "nós") e o estranho (a América Latina, "eles”). O livro de Simpson, certamente, não é o único a concorrer para o desenvolvimento desse processo, mas está inserido em um 
debate atinente ao imaginário norte-americano sobre os latinos e suas culturas. Como aclara a autora em seu estudo, o fenômeno Xuxa reflete aspectos da cultura brasileira - são esses aspectos de que Simpson lança mão para (des)qualificar o fenômeno Xuxa como único e, eu diria também, inferior.

O imaginário norte-americano sobre os povos latinos, portanto, parece ancorar a análise de Simpson. Como observa Rashkin, Xuxa sinaliza exemplo do paradoxo da qualidade de ser loira:

um latino-americano que não é loiro, literal ou figurativamente, é visto como inferior e tem pouca chance de ser bem-sucedido na televisão convencional dos Estados Unidos; já uma estrela latino-americana que é loira é vista como não autêntica, o produto de um público que passou por uma lavagem cerebral por meio de fantasias imperialistas e ideais impossíveis. (RASHKIN, 1999, p. 217).

Dessa forma, Rashkin tem em conta que a recepção de Xuxa pela mídia norte-americana - e, eu acrescentaria, também pela comunidade científica, notadamente pelos pesquisadores dos estudos culturais e da mídia - foi estereotipada, intransigente e reveladora desse imaginário em torno da América Latina, em especial sobre sua cultura popular e o público que a consome.

Em conclusão, a análise de Simpson sobre o fenômeno Xuxa parece desvelar que os indivíduos continuam a ser definidos e classificados, de maneira sutil e algumas vezes paradoxal, pelo sotaque com o qual falam, pela cor de sua pele e de seu cabelo, pelo país e/ou região da qual são oriundos. Os povos latino-americanos continuam sendo rotulados como inferiores, subdesenvolvidos e atrasados, agora acrescida a representação de que esses mesmos países possuem uma população necessitada, dona de baixo índice de escolaridade. Em consequência, essa população é facilmente manipulável por uma indústria dos meios de comunicação de massa superdesenvolvida, que constrói uma cultura popular única, embebida em "fantasias imperialistas e ideais impossíveis". Os autores já não são os mesmos, mas o discurso colonial persiste. Acredito que estudos sobre esses discursos com sabor de colônia se revistam de um papel ainda mais urgente: fazer que essas relaçôes venham à tona no mundo contemporâneo.

\section{Considerações finais}

Com este artigo, intentei analisar o estudo de Simpson sobre o fenômeno Xuxa, alicerçado na premissa do apagamento de qualquer ideal de 
neutralidade e objetividade na produção de conhecimento proposta por Moita Lopes $(2004 ; 2006 ; 2009)$. Dessa forma, denotei que a análise proposta por Simpson mascara um discurso colonialista ao (des)qualificar o fenômeno Xuxa como típico de país pertencente ao terceiro mundo. Por outras palavras, país subdesenvolvido, atrasado, com população definhada por baixo índice de escolaridade, facilmente manipulável pelos meios de comunicação. Meu intuito não foi desmerecer o trabalho da autora, ainda uma das principais referências para aqueles que se interessam em compreender o fenômeno Xuxa no Brasil. Entretanto, entendo que o pesquisador constrói e produz significados sobre o que estuda calcado em sua sócio-história. Portanto, Simpson, na qualidade de professora e pesquisadora da Universidade da Flórida, Estados Unidos, parece reproduzir o imaginário norte-americano sobre a América Latina, especialmente no que toca à sua cultura popular. Os significados que o estudo estampa são reproduzidos como "verdades científicas", a tentar perpetuar um discurso com sabor nitidamente colonialista.

No magistério de Foucault (2012[1979]), o propósito maior de compreender a relação verdade-poder na construção do conhecimento é desvincular o poder da verdade das formas de hegemonia. $\mathrm{O}$ que me parece não ser mais aceitável, portanto, é que a arquitetura do conhecimento continue vindo ao encontro tão só de interesses hegemônicos e, neste particular, basicamente colonialistas, em detrimento de outras versões capazes também elas de agasalhar a "verdade". Sendo assim, oxalá que o enveredar-se pelas estradas desta pesquisa possa ser "[...] um modo de re-inventar a vida social e de nos fazer pensar sobre os significados que construímos em nossas práticas e sobre os sofrimentos que acarretam" (MOITA LOPES, 2009, p. 48).

\section{Referências}

CAMPOS, V. P. M. Querer, poder e conseguir. O processo da socialização para o consumo: o caso Xuxa. 2006. 152 f. Dissertação (Mestrado em Comunicação Social) - Faculdade de Comunicação Social, Pontifícia Universidade Católica do Rio de Janeiro, Rio de Janeiro, 2006.

CURRY, R.; VALDIVIA, A. N. Xuxa at the borders of U. S. TV: checked for gender, race, and national identity. In: MEEHAN, E.; RIORDAN, E. (Eds.). Sex; money. Feminism and Political Economy in the Media. Minneapolis: University of Minnesota Press, 2002. p. 240-256.

FOUCAULT, M. Microfísica do poder. Organização e tradução de Roberto Machado. São Paulo: Graal, 2012. 
LOOMBA, A. Colonialism/Postcolonialism. 2. ed. London; New York: Routledge, 2005.

MILLS, S. Discourse. 2. ed. London; New York: Routledge, 2004.

MOITA LOPES, L. P. (Org.). Por uma lingüistica aplicada indisciplinar. São Paulo: Parábola, 2006.

MOITA LOPES, L. P. Contemporaneidade e construção de conhecimento na área de estudos lingüísticos. Scripta, Belo Horizonte, v. 7, n. 14, p. 159-171, $1^{\circ}$ semestre 2004.

MOITA LOPES, L. P. Lingüística aplicada como lugar de construir verdades contingentes: sexualidades, ética e política. Gragoatá, Niterói, n. 27, p. 33-50, 2o semestre 2009.

RASHKIN, E. Xuxa S. A.: The Queen of Rede Globo in the Age of Transnational Capitalism. In: KINDER, M. (Ed.). Kids' Media Culture. Durham; London: Duke University Press, 1999. p. 204-220.

SAID, E. W. Orientalism. London: Routledge, 1978.

SIMPSON, A. Xuxa. The Mega-Marketing of Gender, Race, and Modernity. Philadelphia: Temple University Press, 1993.

SINVAL, M. J. F. M. $O$ " $X$ " da questão. O fenômeno Xuxa e a construção das crianças com o "X". 2010. 84 f. Dissertação (Mestrado em Comunicação e Semiótica) - Faculdade de Comunicação Social, Pontifícia Universidade Católica de São Paulo, São Paulo, 2010.

VALDIVIA, A. N.; CURRY, R. Xuxa at the Borders of Global TV: The Institutionalization and Marginalization of Brazil's Blonde Ambition. Camera Obscura, n. 38, p. 31-59, 1998.

VALDIVIA, A. N.; CURRY, R. Xuxa!: Can Latin Americans Be Blonde or Can the United States Tolerate a Latin American? In: VALDIVIA, A. N. A Latina in the Land of Hollywood and Other Essays on Media Culture. Tucson: University of Arizona Press, 2000. p. 125-147.

ZOLIN-VESZ, F.; JESUS, D. M. A constituição discursiva da predestinação de Xuxa. Signótica, Goiânia, v. 24, n. 2, p. 323-338, jul./dez. 2012. 